\title{
Extracellular volume imaging by T1 mapping cardiovascular magnetic resonance in patients with clinically suspected myocarditis
}

\author{
Ulf K Radunski ${ }^{*}$, Gunnar Lund ${ }^{2}$, Mandana D Nariman ${ }^{1}$, Bernhard Schnackenburg ${ }^{4}$, Christian Stehning ${ }^{3}$, \\ Gerhard Adam², Stefan Blankenberg ${ }^{1}$, Kai Muellerleile ${ }^{1}$
}

From 16th Annual SCMR Scientific Sessions

San Francisco, CA, USA. 31 January - 3 February 2013

\section{Background}

Assessing the extracellular volume fraction (ECV) by T1 mapping is a promising cardiovascular magnetic resonance (CMR) approach to quantify myocardial injury. This study evaluated ECV imaging by T1 mapping CMR in patients with clinically suspected myocarditis.

\section{Methods}

T1 mapping was implemented into a standard CMR protocol in 113 patients with clinically suspected myocarditis at 1.5 Tesla. T1 quantification was performed for ECV -calculation using the modified Look-Locker inversionrecovery (MOLLI) sequence on three short-axes before and 15 minutes after administration of $0.075 \mathrm{mmol} / \mathrm{kg}$ gadolinium BOPTA, respectively. Global left ventricular ECV was calculated from T1 maps generated with a dedicated plug-in written for the OsiriX software: relaxation rates $(1 / \mathrm{T} 1=\mathrm{R} 1)$ were calculated for myocardium and blood pool. The difference in R1 between pre- and post contrast media was calculated as $\triangle \mathrm{R} 1$. Myocardial ECV was then estimated using the formula: $\mathrm{ECV}=1$-hematocrit * $\left(\Delta \mathrm{R} 1_{\text {myocardium }} / \Delta \mathrm{R} 1_{\text {blood pool }}\right)$. Figure 1 demonstrates an example for T1 maps pre- and post contrast media in comparison with standard black-blood T2 STIR and late gadolinium enhancement (LGE) images.

\section{Results}

The final study population consisted of 96 patients after exclusion of 17 patients with concomitant coronary artery disease or severe left ventricular hypertrophy. Current clinical and CMR criteria identified 64 (67\%) patients with

${ }^{1}$ University Heart Center Hamburg, Hamburg, Germany

Full list of author information is available at the end of the article myocarditis and $32(33 \%)$ patients without myocarditis in the final study population. Global ECV was significantly larger in patients with myocarditis compared to patients without myocarditis ( $35 \pm 6$ vs. $30 \pm 5 \%$, $\mathrm{p}<0.0001)$. Furthermore, global ECV was significantly larger in patients with focal scar compared to patients without focal scar on LGE CMR ( $35 \pm 5$ vs. $33 \pm 7 \%, \mathrm{p}<0.05)$. ROC analysis revealed a sensitivity of $73 \%$ and a specificity $67 \%$ to identify patients with myocarditis using a global ECV $\geqq 32 \%$ cutoff $($ AUC $=0.76, p<0.0001)$.

\section{Conclusions}

Patients with myocarditis are characterized by an increased global ECV. Assessing global ECV by T1 mapping has great potential to improve the diagnostic and prognostic impact of CMR in patients with clinically suspected myocarditis.

\section{Funding}

Orlovic Foundation.

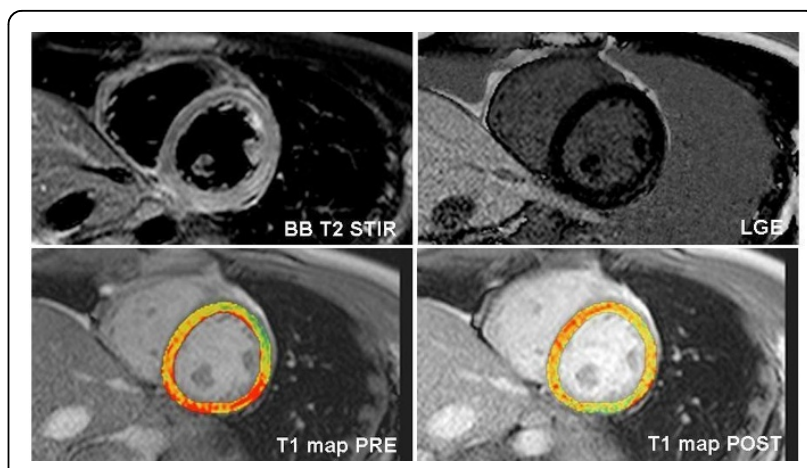

Figure 1 


\section{Author details}

${ }^{1}$ University Heart Center Hamburg, Hamburg, Germany. ${ }^{2}$ Diagnostic and

Interventional Radiology, University Medical Center Hamburg-Eppendorf,

Hamburg, Germany. ${ }^{3}$ Philips Research Hamburg, Hamburg, Germany. ${ }^{4}$ Philips

Healthcare Hamburg, Hamburg, Germany.

Published: 30 January 2013

doi:10.1186/1532-429X-15-S1-P116

Cite this article as: Radunski et al.: Extracellular volume imaging by $\mathrm{T} 1$

mapping cardiovascular magnetic resonance in patients with clinically

suspected myocarditis. Journal of Cardiovascular Magnetic Resonance 2013

15(Suppl 1):P116.

Submit your next manuscript to BioMed Central and take full advantage of:

- Convenient online submission

- Thorough peer review

- No space constraints or color figure charges

- Immediate publication on acceptance

- Inclusion in PubMed, CAS, Scopus and Google Scholar

- Research which is freely available for redistribution

Submit your manuscript at www.biomedcentral.com/submit 